\title{
COEFFICIENT SUBRINGS OF CERTAIN LOCAL RINGS WITH PRIME-POWER CHARACTERISTIC
}

\author{
TAKAO SUMIYAMA
}

\author{
Department of Mathematics \\ Aichi Institute of Technology \\ Yakusa-chô, Toyota \\ 470-03 Japan
}

(Received October 13, 1993 and in revised form September 15, 1994)

\begin{abstract}
If $R$ is a local ring whose radical $J(R)$ is nilpotent and $R / J(R)$ is a commutative field which is algebraic over $G F(p)$, then $R$ has at least one subring $S$ such that $S=\cup_{i=1}^{\infty} S_{l}$, where each $S_{1}$ is isomorphic to a Galois ring and $S / J(S)$ is naturally isomorphic to $R / J(R)$. Such subrings of $R$ are mutually isomorphic, but not necessarily conjugate in $R$.
\end{abstract}

KEY WORDS AND PHRASES: Coefficient ring, Galois ring, local ring, Szele matrix. 1991 AMS SUBJECT CLASSIFICATION CODES: 16L30, 16P10, 16D70

\section{INTRODUCTION}

Let $p$ be a fixed prime. For any positive integers $n$ and $r$, there exists up to isomorphism a unique $r$-dimensional separable extension $G R\left(p^{n}, r\right)$ of $\mathbf{Z} / p^{n} \mathbf{Z}$, which is called the Galois ring of characteristic $p^{n}$ and rank $r$ (see [9, p. 293, Theorem XV.2]). This ring was first noticed by Krull [8], and was later rediscovered by Janusz [6] and Raghavendran [12].

By Wedderburn-Malcev theorem (see, for instance, [4, p. 491]), if $R$ is a finite dimensional algebra over a field $K$ such that $\bar{R}=R / J(R)$ is a separable algebra over $K$, then $R$ contains a semisimple subalgebra $S$ such that $R=S \oplus J(R)$ (direct sum as vector spaces). Such subalgebras of $R$ are conjugate each other.

Concerning the case $R$ is not an algebra over a field, Raghavendran [12, Theorem 8], Clark [3] and Wilson [17, Lemma 2.1] have proved the following: If $R$ is a finite local ring with characteristic $p^{n}$ whose residue field is $G F\left(p^{r}\right)$, then $R$ contains a subring $S$ such that $S$ is isomorphic to $G R\left(p^{n}, r\right)$ (hence $R=S+J(R)$ ). Such a subring $S$ of $R$ is called a coefficient ring of $R$. Coefficient rings of $\mathrm{R}$ are conjugate each other. We can embed $R$ to a ring of Szele matrices over $S$ (see $\S 1$ ).

If $R$ is a finite ring of characteristic $p^{n}$, then $R$ contains a subring $T$ (unique up to isomorphism) such that (1) $R=T \oplus N$ (as abelian groups), where $N$ is an additive subgroup of $J(R)$, (2) $T$ is a direct sum of matrix rings over Galois rings, (3) $J(T)=T \cap J(R)=p T$, and (4) $R=T+J(R)$.

The purpose of this paper is to extend these results to certain rings which are not necessarily finite. 1.

In what follows, when $S$ is a set, $|S|$ will denote the cardinal number of $S$. When $A$ is a ring, for any subset $S$ of $A,\langle S\rangle$ denotes the subring of $A$ generated by $S$. A ring $A$ is called locally finite if any finite subset of $A$ generates a finite subring. When $A$ is a ring with 1 , for $B$ to be called a subring of $A, B$ must contain 1. Let $J(A)$ denote the Jacobson radical of $A, A u t(A)$ the automorphism group of $A$, and $(A)_{n \times n}$ the ring of $n \times n$ matrices having entries in $A$. If $A \ni 1, A^{*}$ denotes the group of units of $A$. For $a \in A^{*}, o(a)$ denotes the multiplicative order of $a$.

The Galois ring $G R\left(p^{n}, r\right)$ is characterized as a ring isomorphic to $\left(\mathbf{Z} / p^{n} \mathbf{Z}\right)[X] /\left(f(X)\left(\mathbf{Z} / p^{n} \mathbf{Z}\right)[X]\right)$, where $f(X) \in\left(\mathbf{Z} / p^{n} \mathbf{Z}\right)[X]$ is a monic polynomial of degree $r$, and is irreducible modulo $p \mathbf{Z} / p^{n} \mathbf{Z}$ (see [7, Chapter XVI]). By [12, Proposition 1], any subring of $G R\left(p^{n}, r\right)$ is isomorphic to $G R\left(p^{n}, s\right)$, where 
$s$ is a divisor of $r$. Conversely, if $s$ is a divisor of $r$, then there is a unique subring of $G R\left(p^{n}, r\right)$ which is isomorphic to $G R\left(p^{n}, s\right)$.

The following lemma is easily deduced from $[16$, Theorem $3(\mathrm{I})]$ and its proof.

LEMMA 1.1. Let $R$ be a finite local ring of characteristic $p^{n}$ whose residue field is $G F\left(p^{r}\right)$. If $a \in R^{*}$ satisfies $o(a)=p^{r}-1$, then the subring $\langle a\rangle$ of $R$ is isomorphic to $G R\left(p^{n}, r\right)$.

A ring $R$ will be called an IG-ring if there exists a sequence $\left\{R_{t}\right\}_{1=1}^{\infty}$ of subrings of $R$ such that $R_{l} \subset R_{\imath+1}, R_{l} \cong G R\left(p^{n}, r_{l}\right)(i \geq 1)$ and $R=\cup_{i=1}^{\infty} R_{l}$, where $\left\{r_{l}\right\}_{i=1}^{\infty}$ is a sequence of positive integers such that $r_{1} \mid r_{1+1}(i \geq 1)$. If $R$ is an IG-ring described above, then $R_{1}$ is the only subring of $R$ which is isomorphic to $G R\left(p^{n}, r_{1}\right)$. So we can write $R=\cup_{i=1}^{\infty} G R\left(p^{n}, r_{1}\right)$.

Let $p$ be a prime, $n$ a positive integer and $1=r_{1} \leq r_{2} \leq \ldots$ an infinite sequence of positive integers such that $r_{l} \mid r_{1+1}$. By the fact we observed above, there exists a natural embedding $\mathfrak{\imath}_{t}^{\prime+1}: G R\left(p^{n}, r_{\imath}\right) \rightarrow G R\left(p^{n}, r_{\imath+1}\right)$ for each $i \geq 1$. Let us put $\mathfrak{l}_{t}^{\prime}=i d_{G R\left(p^{n}, r_{1}\right)}$ and $\mathfrak{l}_{t}^{\prime}=\mathfrak{l}_{,-1}^{\prime} \circ \mathfrak{l}_{j-2}^{\prime-1} \circ \ldots \circ \mathfrak{l}_{1}^{\prime+1}$ for $1 \leq i \leq j$. Then we see that $\left\{G R\left(p^{n}, r_{1}\right), \mathfrak{l}_{l}^{\prime}\right\}$ is an inductive system. The $\operatorname{ring} R=\lim _{\rightarrow} G R\left(p^{n}, r_{l}\right)$ is an IG-ring. Conversely, any IG-ring can be constructed in this way. An IG-ring $R=\cup_{i=1}^{\infty} G R\left(p^{n}, r_{1}\right)$ is a Galois ring if $|R|$ is finite. When $A$ is a ring with 1, a subring $S$ of $A$ is called an IG-subring of $A$ if $S$ is an IG-ring.

PROPOSITION 1.2. Let $R=\cup_{i=1}^{\infty} G R\left(p^{n}, r_{t}\right)$ be an IG-ring. Then:

(I) $\quad R$ is a commutative local ring with radical $J(R)=p R$. The residue field $R / p R$ is $\cup_{i=1}^{\infty} G F\left(p^{r}\right)$.

(II) If $e$ is a positive integer such that $1 \leq e \leq n$, then $R / p^{e} R$ is naturally isomorphic to the IG-ring $\cup_{i=1}^{\infty} G R\left(p^{e}, r_{i}\right)$.

(III) $R$ is a proper homomorphic image of a discrete valuation ring whose radical is generated by $p$.

(IV) Any ideal of $R$ is of the form $p^{e} R(0 \leq e \leq n)$.

(V) $R$ is self-injective.

(VI) $\operatorname{Aut}(R) \cong \lim _{\leftarrow} \operatorname{Aut}\left(G R\left(p^{n}, r_{\imath}\right)\right) \cong \lim _{\leftarrow} \operatorname{Aut}\left(G F\left(p^{r_{1}}\right)\right) \cong \operatorname{Aut}\left(\cup_{i=1}^{\infty} G F\left(p^{r_{1}}\right)\right)$.

PROOF. (I) and (II). For each $i \geq 1$,

$$
0 \rightarrow p^{e} G R\left(p^{n}, r_{l}\right) \rightarrow G R\left(p^{n}, r_{l}\right) \rightarrow G R\left(p^{e}, r_{\iota}\right) \rightarrow 0
$$

is an exact sequence of $G R\left(p^{n}, r_{1}\right)$-modules. So we get the result by [2, Chapitre $2, \S 6, \mathrm{n}^{\circ} 6$, Proposition 8].

(III) Let us put $K=\cup_{i=1}^{\infty} G F\left(p^{r_{t}}\right)$. Let $W_{n}(K)$ be the ring of Witt vectors over $K$ of length $n$ (see [15, Chapter II, §6] or [5, Kapitel II, §10.4]. By (I) and [5, Kapitel II, §10.4], both $R$ and $W_{n}(K)$ are elementary complete local rings (in [14], elementare vollständige lokale Ringe) of characteristic $p^{n}$ whose residue fields are $K$. Since an elementary complete local ring is uniquely determined by its characteristic and residue field (see [14, Anhang 2]), we see that $R$ is isomorphic to $W_{n}(K)$. Let $W(K)$ be the ring of Witt vectors over $K$ of infinite length. By [7, Chapter V, §7], $W(K)$ is a discrete 
valuation ring whose radical is generated by $p$. Since $W(K)$ is the projective limit of $\left\{W_{n}(K)\right\}_{i=1}^{\infty}$ (see [15, Chapter II, §6]), $W_{n}(K)$ is a homomorphic image of $W(K)$.

(IV) If $R$ is a discrete valuation ring with radical $p R$, then any ideal of $R$ is of the form $p^{j} R(j \geq 0)$, so the result is clear from (III).

(V) Clear from (III), since any proper homomorphic image of a principal ideal domain is self-injectıve.

(VI) Immediate by [9, p. 294, Corollary XV.3].

Let $\left\{r_{l}\right\}_{l=1}^{\infty}$ be an infinite sequence of positive integers such that $r_{1}=1$ and $r_{l} \mid r_{l+1}(l \geq 1)$, and $S=\cup_{l=1}^{\infty} G R\left(p^{n}, r_{l}\right)$ be an IG-ring of characteristic $p^{n}$. Let $n=n_{1} \geq n_{2} \geq \ldots \geq n_{t}$ be a nonincreasing sequence of positive integers. Let us put $S_{J}=\cup_{l=1}^{\infty} G R\left(p^{n_{j}}, r_{l}\right)$ for $1 \leq j \leq t$. Let $\phi_{j}: S \rightarrow S$, be the natural homomorphism followed by the isomorphism $S / p^{n} S \cong S$, of Proposition 1.2 (II). Let us put $U\left(S ; n_{1}, n_{2}, \ldots, n_{t}\right)=\left\{\left(\alpha_{t \jmath}\right) \in(S)_{t \times t} \mid \alpha_{\imath j} \in p^{n_{j}-n_{t}} S\right.$ if $\left.i>j\right\}$. It is easy to see that $U\left(S ; n_{1}, n_{2}, \ldots, n_{t}\right)$ forms a subring of $(S)_{t \times t}$. Let $M\left(S ; n_{1}, n_{2}, \ldots, n_{t}\right)$ denote the set of all $t \times t$ matrices $\left(a_{t \jmath}\right)$, where $a_{i j} \in S_{j}$, and $a_{i} \in p^{n_{1}-n_{t}} S$, for $i>j$. Let $\Phi$ be the mapping of $U\left(S ; n_{1}, n_{2}, \ldots, n_{t}\right)$ onto $M\left(S ; n_{1}, n_{2}, \ldots, n_{t}\right)$ defined by $\left(\alpha_{\imath \jmath}\right) \mapsto\left(a_{\imath \jmath}\right)$ where $a_{\imath \jmath}=\phi_{J}\left(\alpha_{\imath \jmath}\right)$. It is easy to check that addition and multiplication in $M\left(S ; n_{1}, n_{2}, \ldots, n_{t}\right)$ can be defined by stipulating that $\Phi$ preserves addition and multiplication. Following [17], we call $M\left(S ; n_{1}, n_{2}, \ldots, n_{t}\right)$ a ring of Szele matrices over $S$.

LEMMA 1.3. (cf. [17, Lemma 2.1]) Let $R$ be a ring with 1 which contains an IG-subring $S$ of characteristic $p^{n}$. If $R$ is finitely generated as a left $S$-module, then there exists a nonincreasing sequence $n=n_{1} \geq n_{2} \geq \ldots \geq n_{t}$ of positive integers such that $R$ is isomorphic to a subring of $M\left(S ; n_{1}, n_{2}, \ldots, n_{t}\right)$.

PROOF. By Proposition 1.2 (V), there exists a submodule $N$ of $R$ such that $R=S \oplus N$ as left $S$-modules. By Proposition 1.2 (III), there are a discrete valuation ring $W$ and a homomorphism $\phi$ of $W$ onto $S$. By defining

$$
a y=\phi(a) y(a \in W, y \in N),
$$

$N$ is a finitely generated $W$-module. Since a finitely generated module over a principal ideal domain is a direct sum of cyclic modules, there exist $y_{1}, y_{2}, \ldots, y_{\mathrm{s}} \in N$ such that $N=\oplus_{1=1}^{s} W y_{t}$. Let $t=s+1, x_{1}=1$ and $x_{t}=y_{t-1}(2 \leq i \leq t)$. Then we get $R=\oplus_{t=1}^{t} S x_{t}$. Let $S x_{t} \cong S / p^{n} S$ as $S$-modules $\left(n_{1}=n\right)$. Without loss of generality, we may assume $n_{1} \geq n_{2} \geq \ldots \geq n_{t}$. For each $a \in R$, we can write

$$
x_{i} a=\sum_{j=1}^{t} \alpha_{\imath \jmath} x_{j}\left(\alpha_{i j} \in S\right) \text {. }
$$

Since

$$
0=p^{n_{i}} x_{t} a=\sum_{j=1}^{n} p^{n_{i}} \alpha_{i j} x_{J},
$$

by Proposition 1.2 (IV), $\alpha_{i j} \in p^{n_{j}-n_{i}} S$ if $i>j$. As $\alpha_{i j}$ is uniquely determined modulo $p^{n_{i}} S$ by $a$, we can define $\psi: R \rightarrow M\left(S ; n_{1}, n_{2}, \ldots, n_{t}\right)$ by $a \mapsto\left(\psi_{J}\left(\alpha_{t}\right)\right)$. It is easy to see that $\psi$ is an injective ring homomorphism.

2.

Let $G$ be a group, and $N$ a normal subgroup of $G$. Let $\rho: G \rightarrow H=G / N$ be the natural homomorphism. A monomorphism $\lambda: H \rightarrow G$ will be called a right inverse of $\rho$ if $\rho \circ \lambda=i d_{H}$. If $\lambda$ is a right inverse of $\rho$, then $G$ is a semidirect product of $N$ and $\lambda(H)$.

The following lemma is a variation of Schur-Zassenhaus theorem [13, Chapter 9, 9.1.2]. 
LEMMA 2.1. Let $G$ be a group, and $N$ a normal subgroup of $G$. Let $\rho: G \rightarrow H=G / N$ be the natural homomorphism. Assume that $N$ is locally finite, and there exists a sequence $\left\{H_{l}\right\}_{i=1}^{\infty}$ of finite subgroups of $H$ such that $H_{i} \subset H_{i+1}(i \geq 1), \cup_{i=1}^{\infty} H_{t}=H$ and, for any $a \in N$ and any $i \geq 1, o(a)$ and $\left|H_{i}\right|$ are coprıme. Then:

(I) There exists a right inverse $\lambda: H \rightarrow G$ of $\rho$.

(II) If, for some $m \geq 1$, there exists a monomorphism $\mu^{\prime}: H_{m} \rightarrow G$ such that $\rho \circ \mu^{\prime}=i d_{H_{m}}$, then there exists a right inverse $\mu: H \rightarrow G$ of $\rho$ such that $\left.\mu\right|_{H_{m}}=\mu^{\prime}$.

(III) There exists a unique right inverse of $\rho$ If and only if $G$ is a nilpotent group.

(IV) If $\mu^{\prime}: H_{m} \rightarrow G$ and $\mu^{\prime \prime}: H_{m} \rightarrow G$ are monomorphısms such that $\rho \circ \mu^{\prime}=\rho \circ \mu^{\prime \prime}=i d_{H_{m}}$, then $\mu^{\prime}\left(H_{m}\right)$ and $\mu^{\prime \prime}\left(H_{m}\right)$ are conjugate in G.

PROOF. (I) For each $x \in H_{1}$, we can choose an element $g_{\text {r }}$ of $G$ such that $\rho\left(g_{r}\right)=x$. As $G$ is locally finite (see [13, Chapter 14, 14.3.1]), the subgroup $G_{1}$ of $G$ generated by $\left\{g_{\mathrm{r}}\right\}_{\mathrm{r} \in H_{1}}$ is finite, and $\left.\rho\right|_{G_{1}}$ is a homomorphism of $G_{1}$ onto $H_{1}$. Let us put $N_{1}=\operatorname{Ker}\left(\left.\rho\right|_{G_{1}}\right)$. Since $\left|N_{1}\right|$ and $\left|H_{1}\right|$ are coprime, by Schur-Zassenhaus theorem [13, Chapter 9, 9.1.2], there exists a right inverse $\lambda_{1}: H_{1} \rightarrow G_{1}$ of $\left.\rho\right|_{G_{1}}$. Next, let $\left\{g_{1}{ }^{\prime}\right\}_{v \in H_{2}}$ be a set of elements of $G$ such that $\rho\left(g_{1}{ }^{\prime}\right)=y$ for any $y \in H_{2}$, and $\left\{g_{r}\right\}_{r \in H_{1}} \subset\left\{g_{1}{ }^{\prime}\right\}_{\imath \in H_{2}}$. Let $G_{2}$ be the finite subgroup of $G$ generated by $\left\{g_{1}{ }^{\prime}\right\}_{\vee \in H_{2}}$. Then $\left.\rho\right|_{G_{2}}$ is a homomorphism of $G_{2}$ onto $H_{2}$. By [13, Chapter 9, 9.1.3], there exists a complement subgroup $L$ of $N_{2}=\operatorname{Ker}\left(\left.\rho\right|_{G_{2}}\right)$ in $G_{2}$ such that $L \supset \lambda_{1}\left(H_{1}\right)$. The mapping $\lambda_{2}: H_{2} \rightarrow G_{2}$ defined by $H_{2}=G_{2} / N_{2} \ni b N_{2} \mapsto b(b \in L)$ is a right inverse of $\left.\rho\right|_{G_{2}}$. For any $a \in H_{1}, \lambda_{2}(a)^{-1} \lambda_{1}(a) \in N_{2} \cap L=\{1\}$, hence we see $\left.\lambda_{2}\right|_{H_{1}}=\lambda_{1}$. Continuing this process inductively, we get a sequence $G_{1} \subset G_{2} \subset \ldots$ of finite subgroups of $G$ and a sequence $\left\{\lambda_{t}\right\}_{t=1}^{\infty}$ of right inverses $\lambda_{t}: H_{t} \rightarrow G_{t}$ of $\left.\rho\right|_{G_{1}}$ such that $\left.\lambda_{J}\right|_{H_{t}}=\lambda_{t}$ for any $1 \leq i \leq j$. Then $\lambda=\lim _{\rightarrow} \lambda_{1}: H=\cup_{1=1}^{\infty} H_{1} \rightarrow G$ is a right inverse of $\rho$.

(II) can also be proved in the same way by starting from $\mu^{\prime}: H_{m} \rightarrow \mu^{\prime}\left(H_{m}\right)$.

(III) Assume that $\lambda: H \rightarrow G$ is the unique right inverse of $\rho$. Then $G$ is a semidirect product of $N$ and $\lambda(H)$. We shall show that this is the direct product. Suppose that there exist $c \in N$ and $z \in H$ such that $c \lambda(z) \neq \lambda(z) c$. Let us define $\mu: H \rightarrow G$ by $\mu(b)=z^{-1} \lambda(b) z$. Then $\mu$ is a right inverse of $\rho$ different from $\lambda$, which contradicts our hypothesis. So $G$ is the direct product of $N$ and $\lambda(H)$. Hence $G$ is nilpotent.

Conversely, let us suppose that $G$ is nilpotent, and $\lambda$ and $\mu$ are right inverses of $\rho$. For each $i \geq 1$, let $G_{\imath}$ be the subgroup of $G$ generated by $\lambda\left(H_{t}\right) \cup \mu\left(H_{l}\right)$. Then $\left.\rho\right|_{G_{l}}$ is a homomorphism of $G_{l}$ onto $H_{\imath}$. Both $\lambda\left(H_{t}\right)$ and $\mu\left(H_{t}\right)$ are complement subgroups for $N_{t}=\operatorname{Ker}\left(\left.\rho\right|_{G_{t}}\right)$ in $G_{l}$. Since $G_{t}$ is a finite nilpotent group, for each prime divisor $q$ of $\left|G_{l}\right|, G_{l}$ contains a unique $q$-Sylow subgroup. Each $G_{l}$ is the direct product of such Sylow subgroups. As $\left|H_{\imath}\right|$ and $\left|N_{\imath}\right|$ are coprime, we have $\lambda\left(H_{\imath}\right)=\mu\left(H_{\imath}\right)$. So $\left.\lambda\right|_{H_{1}}=\left.\mu\right|_{H_{i}}$. Since this holds for each $i \geq 1$, we see $\lambda=\mu$.

(IV) Let $L$ be the finite subgroup of $G$ generated by $\mu^{\prime}\left(H_{m}\right) \cup \mu^{\prime \prime}\left(H_{m}\right)$. Then $\left.\rho\right|_{L}$ is a homomorphism of $L$ onto $H_{m}$. Since $\left|\operatorname{Ker}\left(\left.\rho\right|_{L}\right)\right|=|N \cap L|$ and $\left|H_{m}\right|$ are coprime, by Schur-Zassenhaus theorem, $\mu^{\prime}\left(H_{m}\right)$ and $\mu^{\prime \prime}\left(H_{m}\right)$ are conjugate in $L$.

Let $G, N, H$ and $\rho: G \rightarrow H$ be as in Lemma 2.1. We say that $G$ has property (GC) with respect to $N$ if, for any two right inverses $\mu$ and $v$ of $\rho, \mu(H)$ and $v(H)$ are conjugate in $G$. If $H$ is finite, then by Lemma 2.1 (IV), $G$ has the property (GC) with respect to $N$. 
Let $R$ be a ring with 1 . Let $S$ be a subring of $R$, and $I=J(R) \cap S$. The homomorphism of $S / I$ to $R / J(R)$ defined by $a+I \mapsto a+J(R)(a \in S)$ is injective. We shall say that $S / I$ is naturally isomorphic to $R / J(R)$ If this homomorphism is onto. If $S$ is a local subring of a local ring $R$ and if $J(S)$ is nilpotent, then $J(S)=J(R) \cap S$.

Now we shall state main theorems of this section, which generalize the result of R. Raghavendran $[9$, p. 373, Theorem XIX.4].

THEOREM 2.2. Let $R$ be a local ring with radical $M$. Assume that $M$ is nilpotent, and $K=R / M$ is a commutative field of characteristic $p$ ( $p$ a prime) which is algebraic over $G F(p)$. Then there exists an IG-subring $S$ of $R$ such that $S / p S$ is naturally isomorphic to $K$.

PROOF. Since $K$ is algebraic over $G F(p),|K|$ is either finite or countably infinite. So there exists a sequence $\left\{K_{1}\right\}_{t=1}^{\infty}$ of finite subfields of $K$ such that $K_{t} \subset K_{t+1}(i \geq 1)$ and $\cup_{t=1}^{\infty} K_{t}=K$. Let $K_{t}=G R\left(p^{r_{r}}\right)$. The natural homomorphism $\pi: R \rightarrow K$ induces a group homomorphism $\pi^{*}=\left.\pi\right|_{R^{*}}$ of $R^{*}$ onto $K^{*}$. Each $\left(1+M^{t}\right) /\left(1+M^{1+1}\right)$ is isomorphic to the additive group $M^{1} / M^{1+1}$. As $p M^{\prime} \subset M^{1+1}$, the order of each element of $1+M=\operatorname{Ker} \pi^{*}$ is a power of $p$. Furthermore, $K^{*}=\cup_{i=1}^{\infty} K_{i}^{*}$, where $\left|K_{i}^{*}\right|=p^{r_{1}}-1$ is coprime to $p$. So, by Lemma 2.1 (I), there exists a right inverse $\lambda: K^{*} \rightarrow R^{*}$ of $\pi^{*}$. For each $i \geq 1$, let $\alpha_{1}$ be a generator of $K_{1}^{*}$. By Lemma 1.1, the subring $S_{l}=\left\langle\lambda\left(\alpha_{t}\right)\right\rangle$ of $R$ is isomorphic to $G R\left(p^{n}, r_{1}\right)$, where $p^{n}$ is the characteristic of $R$. Consequently, $S=\left\langle\lambda\left(K^{*}\right)\right\rangle=\cup_{t=1}^{\infty} S_{t}$ is an IG-subring of $R$, and $S / p S$ is naturally isomorphic to $K$.

Such a subring $S$ of $R$ stated in Theorem 2.2 will be called a coefficient subring of $R$. When $R$ is a commutative local ring satisfying the assumption of Theorem 2.2, $S$ coincides with the subring described in [11, p. 106, Theorem 31.1].

Let $R, M, S$ and $K=\cup_{i=1}^{\infty} G F\left(p^{r_{1}}\right)$ be as in Theorem 2.2, where $\left\{r_{l}\right\}_{i=1}^{\infty}$ is a sequence of positive integers such that $r_{l} \mid r_{t+1}(i \geq 1)$. Let $p^{n}$ be the characteristic of $R$. Let $S^{\prime}$ be another coefficient subring of $R$. From what was stated in $\S 1, S^{\prime} \cong \cup_{i=1}^{\infty} G R\left(p^{n}, r_{1}\right)$, which is isomorphic to $S$. By Proposition $1.2(\mathrm{~V})$, there exists a left $S^{\prime}$-submodule $N$ of $R$ such that $R=S^{\prime} \oplus N$ as left $S^{\prime}$-modules.

If $\lambda: K^{*} \rightarrow R^{*}$ is a right inverse of $\pi^{*}$, then by the proof of Theorem $2.3, S=\left\langle\lambda\left(K^{*}\right)\right\rangle$ is a coefficient subring of $R$.

We shall show that, if $\lambda$ and $\mu$ are different right inverses of $\pi^{*}$, then $\left\langle\lambda\left(K^{*}\right)\right\rangle \neq\left\langle\mu\left(K^{*}\right)\right\rangle$. Let us suppose $\left\langle\lambda\left(K^{*}\right)\right\rangle=\left\langle\mu\left(K^{*}\right)\right\rangle$ and denote it by $S$. Let $\left\{K_{l}\right\}_{i=1}^{\infty}$ be a sequence of finite subfields of $K$ such that $K_{l} \cong G F\left(p^{r^{\prime}}\right), K_{l} \subset K_{t+1}(i \geq 1)$ and $\cup_{t=1}^{\infty} K_{t}=K$. As $\lambda \neq \mu$, there exist a number $j \geq 1$ and an element $\alpha$ of $K_{\text {, such that }} \lambda(\alpha) \neq \mu(\alpha)$. By Lemma 1.1, both $T=\left\langle\lambda\left(K_{J}^{*}\right)\right\rangle$ and $T^{\prime}=\left\langle\mu\left(K_{,}^{*}\right)\right\rangle$ are isomorphic to $G R\left(p^{n}, r_{\jmath}\right)$. As $S=\cup_{l=1}^{\infty}\left\langle\lambda\left(K_{t}^{*}\right)\right\rangle$, there exists a number $l \geq 1$ such that $T \cup T^{\prime} \subset\left(\lambda\left(K_{l}^{*}\right)\right\rangle$. Since $\left\langle\lambda\left(K_{l}^{*}\right)\right\rangle$ is a Galois ring, $T \cong T^{\prime}$ implies $T=T^{\prime}$. The restriction $\left.\pi\right|_{T^{*}}$ is a homomorphism of $T^{*}$ onto $K_{,}^{*}$. Both $\left.\lambda\right|_{K_{j}^{*}}$ and $\left.\mu\right|_{K_{j}^{*}}$ are right inverses of $\left.\pi\right|_{T^{*}}$, so $T^{*}$ is the direct product of $\lambda\left(K_{,}^{*}\right)$ and $\operatorname{Ker}\left(\left.\pi\right|_{T^{*}}\right)=1+p T$, and is also the direct product of $\mu\left(K_{J}^{*}\right)$ and $1+p T$. As $\left|K_{J}^{*}\right|$ and $|1+p T|$ are coprime, we have $\lambda\left(K_{J}^{*}\right)=\mu\left(K_{J}^{*}\right)$. So there exists some $\beta \in K_{J}^{*}$ such that $\lambda(\alpha)=\mu(\beta)$. Then $\alpha=\pi^{*} \circ \lambda(\alpha)=\pi^{*} \circ \mu(\beta)=\beta$, which means $\lambda(\alpha)=\mu(\alpha)$. This contradicts our choice of $\alpha$.

By making use of Lemma 2.1 (I), we can easily see that, if $S$ is a coefficient subring of $R$, there exists a right inverse $\lambda: K^{*} \rightarrow S^{*}$ of $\pi^{*}$ such that $S=\left\langle\lambda\left(K^{*}\right)\right\rangle$.

Summarizing the above, we obtain the following theorem.

THEOREM 2.3. Let $R$ be a local ring with radical $M$. Assume that $M$ is nilpotent, and $K=R / M$ is a commutative field of characteristic $p$ ( $p$ a prime) which is algebraic over $G R(p)$. Let $\pi^{*}: R^{*} \rightarrow K^{*}$ 
be the group homomorphism induced by the natural ring homomorphism $\pi: R \rightarrow K$. Then:

(I) If $S^{\prime}$ is a coefficient subring of $R$, then there exists a $S^{\prime}$-submodule $N$ of $R$ such that $R=S^{\prime} \oplus N$ as left $S^{\prime}$-modules.

(II) All coefficient subrings of $R$ are isomorphic.

(III) If $\lambda: K^{*} \rightarrow R^{*}$ is a right inverse of $\pi^{*}$, then $S=\left\langle\lambda\left(K^{*}\right)\right\rangle$ is a coefficient subring of $R$. Conversely, if $S$ is a coefficient subring of $R$, then there exists uniquely a right inverse $\lambda: K^{*} \rightarrow R^{*}$ of $\pi^{*}$ such that $S=\left\langle\lambda\left(K^{*}\right)\right\rangle$.

(IV) All coefficient subrings of $R$ are conjugate in $R$ if and only if $R^{*}$ has property (GC) with respect to $1+M$.

With the same notation as in Theorem $2.2, M / M^{2}$ is regarded as a left $K$-space by the operation

$$
\bar{a} \bar{x}=\overline{a x}\left(\bar{a} \in K=R / M, \bar{x} \in M / M^{2}\right) .
$$

THEOREM 2.4. Let $R$ be a local ring with radical $M$. Assume that $M$ is nilpotent, and $K=R / M$ is a commutative field of characteristic $p$ ( $p$ a prime) which is algebraic over $G F(p)$. Let $S$ be a coefficient subring of $R$. Then $R$ is finitely generated as a left $S$-module if and only if $M / M^{2}$ is a finite dimensional left $K$-space. In this case, there exists a finitely generated left $S$-submodule $N$ of $M$ such that $R=S \oplus N$ as left $S$-modules, and there exists a nonincreasing sequence $n_{1} \geq n_{2} \geq \ldots \geq n_{t}$ of positive integers $\left(p^{n_{1}}\right.$ is the characteristic of $R)$ such that $R$ is isomorphic to a subring of $M\left(S ; n_{1}, n_{2}, \ldots, n_{t}\right)$.

PROOF. Assume that $R$ is finitely generated as left $S$-module. Then $R$ is a Noetherian left $S$-module, since $S$ is a Noetherian ring by Proposition 1.2 (IV). As $M$ is a left $S$-submodule of $R, M$ is a finitely generated left $S$-module. This implies that $M / M^{2}$ is a finite dimensional left $K$-space.

Conversely, let us assume that $M / M^{2}$ is a finite dimensional left $K$-space. Let $\omega$ be the nilpotency index of $M$. Let $x_{1}, x_{2}, \ldots, x_{d}$ be elements of $M$ whose images modulo $M^{2}$ form a $K$-basis of $M / M^{2}$. As $S / p S$ is naturally isomorphic to $K$, any element of $y$ of $M$ is written as

$$
y=\sum_{t=1}^{d} a_{1} x_{i}+y^{\prime} \quad\left(a_{i} \in S, y^{\prime} \in M^{2}\right) .
$$

Let

$$
z=\sum_{J=1}^{d} b_{J} x_{j}+z^{\prime} \quad\left(b_{J} \in S, z^{\prime} \in M^{2}\right)
$$

be another element of $M$. Then

$$
y z=\sum_{t, 1=1}^{d} a_{i} x_{i} b_{j} x_{j}+w^{\prime \prime} \quad\left(w^{\prime \prime} \in M^{3}\right) .
$$

Each $x_{i} b$, is written as

$$
x_{\imath} b_{\jmath}=\sum_{k=1}^{d} c_{k \jmath} x_{k}+w_{\imath \jmath}{ }^{\prime} \quad\left(c_{k \jmath \jmath} \in S, w_{\imath \jmath}{ }^{\prime} \in M^{2}\right) .
$$

So we see that any element $v^{\prime}$ of $M^{2}$ can be written as

$$
v^{\prime}=\sum_{t, j=1}^{d} a_{i j} x_{i} x_{j}+v^{\prime \prime} \quad\left(a_{i j} \in S, v^{\prime \prime} \in M^{3}\right) .
$$

Continuing in this way, we see that any element of $M$ is written as an $S$-coefficient linear combination of distinct products of $\omega-1$ or fewer $x_{i}$ 's. So $M$ is a finitely generated left $S$-module. Also $K=R / M$ is a finitely generated left $S$-module, hence $R$ is a finitely generated left $S$-module.

Now suppose that $R$ is finitely generated as left $S$-module. By Theorem 2.3 (I), there exists a finitely generated left $S$-submodule $N^{\prime}$ of $R$ such that $R=S \oplus N^{\prime}$ as left $S$-modules. By Proposition 1.2 (III), there exist a discrete valuation ring $V$ and a homomorphism $\xi$ of $V$ onto $S$. Defining $a y=\xi(a) y\left(a \in V, y \in N^{\prime}\right)$, we can regard $N^{\prime}$ as a left $V$-module. Then there exist $x_{1}, x_{2}, \ldots, x_{t} \in N^{\prime}$ 
such that $N^{\prime}=\oplus_{1=1}^{\prime} V x_{1}=\oplus_{1=1}^{\prime} S x_{1}$. By putting $x_{0}=1$, we get $R=\oplus_{1=0}^{\prime} S x_{1}$. Let $c_{1}, c_{2}, \ldots, c_{t}$ be elements of $S$ such that $\bar{c}_{1}=\bar{x}_{1}$ under the natural homomorphism $\pi: R \rightarrow K$. Let us put $y_{0}=1$ and $y_{1}=x_{1}-c_{1}$ for $1 \leq i \leq t$. Then $y_{i} \in M(1 \leq i \leq t)$ and $R=\oplus_{1=0}^{\prime} S x_{i}=\oplus_{1=0}^{\prime} S y_{1}$. So $N=\oplus_{i=1}^{t} S y_{t}$ has the desired property. The last statement is immediate from Lemma 1.3.

3.

Let $R$ be a local ring described in Theorem 2.2. Then $R$ may have more than one coefficient subring. Concerning this subject, first we can state the following.

THEOREM 3.1. Let $T$ be an IG-ring of characteristic $p^{n}$ different from $G R\left(p^{n}, 1\right)$. Then, for any infinite cardinal number $\chi$, there exists a local ring $R$ such that

(1) $M=J(R)$ is nilpotent,

(2) $K=R / M$ is a commutative field of characteristic $p$ ( $p$ a prime) which is algebraic over $G F(p)$,

(3) coefficient subrings of $R$ are isomorphic to $T$,

(4) all coefficient subrings of $R$ are conjugate in $R$, and

(5) $\chi$ is the number of all coefficient subrings of $R$.

PROOF. Let $T=\cup_{1=1}^{\infty} G R\left(p^{n}, r_{1}\right)$, where $\left\{r_{1}\right\}_{i=1}^{\infty}$ is a sequence of positive integers such that $r_{1} \mid r_{1+1}(i \geq 1)$. Let $K=T / p T$ and $\pi^{\prime}: T \rightarrow K$ be the natural homomorphism. As $K$ is a proper extension of $G F(p)$, there exists an automorphism $\bar{\sigma}$ of $K$ different from $i d_{K}$. Let $\sigma$ be the automorphism of $T$ which induces $\bar{\sigma}$ modulo $p T$ (see Proposition $1.2(\mathrm{VI})$ ). Let $A$ be a set of cardinality $\chi$, and $V=\oplus_{\alpha \in A} T$ be a free $T$-module. The abelian group $T \oplus V$ together with the multiplication

$$
(a, x)\left(a^{\prime}, x^{\prime}\right)=\left(a a^{\prime}, a x^{\prime}+\sigma\left(a^{\prime}\right) x\right)
$$

forms a ring, which we denote by $R$. Let $\pi: R \rightarrow K$ be the homomorphism defined by $(a, x) \mapsto \pi^{\prime}(a)$, and $M=K e r \pi$. As $R / M \cong K$ and $M^{n+1}=0, R$ is a local ring with radical $M$ whose residue field is $K$. By Theorem 2.3 (III), there exists a one-to-one correspondence between the set of all coefficient subrings of $R$ and the set $Y$ of all right inverses of $\pi^{*}=\left.\pi\right|_{R^{*}}: R^{*} \rightarrow K^{*}$.

By the embedding $T \ni a \rightarrow(a, 0) \in R, T$ is regarded as a coefficient subring of $R$. So, by Theorem 2.3 (III), there exists a right inverse $\lambda: K^{*} \rightarrow R^{*}$ of $\pi^{*}$ such that $\left\langle\lambda\left(K^{*}\right)\right\rangle=T$. Since $K=\cup_{i=1}^{\infty} G F\left(p^{r^{r}}\right)$, there exists a number $j \geq 1$ such that $\bar{\sigma}$ is not the identity on $G F\left(p^{r^{\prime}}\right)$. Let $\gamma$ be a generator of $G F\left(p^{r^{\prime}}\right)^{*}$, and $c=\lambda(\gamma)$. It is easy to see that, for any $z \in V, R^{*} \ni h=(c, z)$ is of multiplicative order $p^{r_{J}}-1$. So, for each $z \in V$, we can define a group homomorphism

$\mu_{z}^{\prime}: G F\left(p^{{ }^{\prime}}\right)^{*} \rightarrow R^{*}$ by $\gamma \mapsto(c, z)^{l}$. By Lemma 2.1 (II), we can extend $\mu_{z}^{\prime}$ to $\mu_{z} \in Y$. If $V \ni z_{1}, z_{2}$ and $z_{1} \neq z_{2}$, then $\mu_{21} \neq \mu_{z 2}$. So $|Y| \geq|V|=\chi$.

Let $S$ be a coefficient subring of $R$. We shall show that $S$ is conjugate to $T$. By Theorem 2.3 (III), there exists a right inverse $\lambda^{\prime}: K^{*} \rightarrow R^{*}$ of $\pi^{*}$ such that $S=\left\langle\lambda^{\prime}\left(K^{*}\right)\right\rangle$. Let $\lambda^{\prime}(\gamma)=\left(c^{\prime}, z\right)$, where $c^{\prime} \in T$ and $z \in V$. Let $U$ be the finite subgroup of $R^{*}$ generated by $\lambda(\gamma)$ and $\lambda^{\prime}(\gamma)$. As the restriction $\left.\pi\right|_{U}$ is a homomorphism of $U$ onto $G F\left(p^{r^{\prime}}\right)^{*}$, by Schur-Zassenhaus theorem, there exists $(b, w) \in R^{*}(b \in T, w \in V)$ and an integer $i$ such that $\lambda^{\prime}(\gamma)=(b, w)^{-1} \lambda(\gamma)(b, w)$. Then, $\left(c^{\prime}, z\right)=(b, w)^{-1}\left(c^{\prime}, 0\right)(b, w)$, which implies $c^{\prime}=c^{\prime} . \quad$ As $\pi^{\prime}\left(c^{\prime}\right)=\pi\left(\lambda^{\prime}\left(c^{\prime}\right)\right)=\gamma=\pi(\lambda(\gamma))=\pi^{\prime}(c)$, so $c^{\prime}=c$ and $\lambda^{\prime}(\gamma)=(c, z)$. Let $x=\{c-\sigma(c)\}^{-1} z$. Suppose that $\alpha \in K$ satisfies $\alpha^{m}=\gamma$ for some integer $m$. Let $\lambda(\alpha)=a$. Then, by the same reason as above, we can write $\lambda^{\prime}(\alpha)=(a, y)$ for some $y \in V$. 
As

$$
(c, z)=\lambda^{\prime}(\gamma)=\lambda^{\prime}\left(\alpha^{m}\right)=(a, y)^{m}=\left(a^{m},\left\{a^{m}-(\sigma(a))^{m}\right\}\{a-\sigma(a)\}^{-1} y\right),
$$

we get $c=a^{\prime \prime}$ and $z=\{c-\sigma(c)\}\{a-\sigma(a)\}^{-1} y$. So $(1, x) \lambda^{\prime}(\alpha)=(a, y+\sigma(a) x)=(a, a x)=\lambda(\alpha)(1, x)$. As $K^{*}$ is the union of cyclic subgroups generated by such $\alpha$ which contain $G F\left(p^{\prime}\right)^{*}$ (generated by $\gamma$ ), this proves $S=\left\langle\lambda^{\prime}\left(K^{*}\right)\right\rangle=(1, x)^{-1} T(1, x)$. So $|Y|$, the number of all coefficient subrings of $R$. does not exceed $\chi$. As we have seen $|Y| \geq \chi$, we get $|Y|=\chi$.

Next we shall consider the uniqueness of coefficient subrings.

A finite local ring $T$ is called of type ( 1 ) if $T$ is generated by two units $a$ and $b$ such that

(1) $a b \neq b a$,

(2) $a-b \in J(T)$, and

(3) $o(a)=o(b)=|T / J(T)|-1$.

If $T$ is a finite local ring of type ( 1 ), then $T^{*}$ is not a nilpotent group. For, let us suppose that $T$ is a finite local ring of type (1). Let $a$ and $b$ be generators of $T$ satisfying (1)-(3). Let $A$ and $B$ be cycic subgroups of $T^{*}$ generated by $a$ and $b$ respectively. Let $K=T / J(T)=G F\left(p^{r}\right)$. Then $|A|=|B|=p^{r}-1$ is coprime to $|J(T)|$. If $T^{*}$ is nilpotent, then $A=B$, as both $A$ and $B$ are complement subgroups of $1+J(T)$ in $T^{*}$. This contradicts (1), so we see that $T^{*}$ is not nilpotent.

THEOREM 3.2. Let $R$ be a local ring with radical $M$. Assume that $M$ is nilpotent, and $K=R / M$ is a commutative field of characteristic $p$ ( $p$ a prime) which is algebraic over $G F(p)$. Then the following are equivalent.

(i) $R$ has a unique coefficient subring.

(ii) $R^{*}$ is a nilpotent group.

(iii) $R^{*}$ is isomorphic to the direct product of $K^{*}$ and $1+M$.

(iv) $R^{*}$ has no finite local subring of type (1).

PROOF. (i) $\Leftrightarrow$ (ii). Clear from Lemma 2.1 (III) and Theorem 2.3 (III).

(i) $\Rightarrow$ (iii). Let $\pi^{*}=\left.\pi\right|_{R^{*}}: R^{*} \rightarrow K^{*}$ be the group homomorphism induced by the natural homomorphism $\pi: R \rightarrow K$. Since $R$ has a unique coefficient subring, by Theorem 2.3 (III), there exists a unique right inverse $\lambda$ of $\pi^{*}$. Then $R^{*}$ is a semidirect product of $1+M$ and $K^{*}$. Let $z$ be any fixed element of $1+M$. The mapping $\mu: K^{*} \rightarrow R^{*}$ defined by $K^{*} \ni \alpha \mapsto z^{-1} \lambda(\alpha) z$ is a right inverse of $\pi^{*}$, so $\mu=\lambda$ by our hypothesis. This implies that each element of $\lambda\left(K^{*}\right)$ commutes with each element of $1+M$. Hence $R^{*}$ is the direct product of $1+M$ and $\lambda\left(K^{*}\right)$.

(iii) $\Rightarrow$ (iv). Let us suppose that $R$ contains a finite local subring $U$ of type (1). By the proof of [10, Lemma 1], $1+M$ is a nilpotent group. If $R^{*}$ is isomorphic to the direct product of $K^{*}$ and $1+M$, then $R^{*}$ is nilpotent. So $U^{*}$ is nilpotent, which is a contradiction.

(iv) $\Rightarrow$ (i). Assume that $R$ has at least two different coefficient subrings. Then there exist at least two different right inverses $\lambda$ and $\mu$ of $\pi^{*}$. Let $\left\{K_{l}\right\}_{l=1}^{\infty}$ be a sequence of finite subfields of $K$ such that $K_{l} \subset K_{t+1}$ and $\cup_{i=1}^{\infty} K_{l}=K$. There exists a number $j$ such that $\left.\lambda\right|_{K_{j}^{*}} \neq\left.\mu\right|_{K_{,}^{*}}$. Let $\gamma$ be a generator of $K_{J}^{*}$. Then the subring $\langle\lambda(\gamma), \mu(\gamma)\rangle$ of $R$ is a finite local ring of type (l).

4.

From [9, p. 373, Theorem XIX.4 (b)] and the proof of Theorem 3.1, one may expect that, in Theorem 2.3 , any two coefficient subrings of $R$ are always conjugate. However, from the following example, we see that this is incorrect. 
Let $K=\cup_{i=1}^{\infty} G F\left(p^{r_{1}}\right)$, where $\left\{r_{i}\right\}_{1=1}^{\infty}$ is a strictly increasing sequence of positive integers such that $r_{1} \mid r_{1+1}(i \geq 1)$. Let $\left\{\sigma_{1}\right\}_{1=1}^{\infty}$ be automorphisms of $K$ such that $\sigma_{1}$ is not the identity on $G F\left(p^{r_{1}}\right)(i \geq 1)$ and, for $j<i, \sigma_{l}$ is the identity on $G F\left(p^{{ }^{\prime}}\right)$. Let $V=\oplus_{i=1}^{\infty} K x_{l}$ be a left $K$-vector space with basis $\left\{x_{i}\right\}_{i=1}^{\infty}$. We can regard $V$ as a $(K, K)$-bimodule by defining

$$
\left(\Sigma_{l} c_{1} x_{t}\right) a=\sum_{l} c_{1} \sigma_{t}(a) x_{1}\left(\sum_{1} c_{1} x_{1} \in V, a \in K\right) .
$$

The abelian group $R=K \oplus V$ together with the multiplication

$$
(a, y)(b, z)=(a b, a z+y b)(a, b \in K, y, z \in V)
$$

forms a local ring with radical $M=(0, V)$, which satisfies the assumption of Theorem 2.3. The homomorphism $\pi: R \rightarrow K$ defined by $(a, x) \mapsto a$ gives the isomorphism $R / M \cong K$. The subring $S=\{(a, 0) \mid a \in K\}$ of $R$ is a coefficient subring of $R$.

For each $i \geq 1$, let $\gamma_{1}$ be a generator of $G F\left(p^{r_{1}}\right)^{*}$. Then we can write $\gamma_{1}=\gamma_{1+1}^{m_{1}}$ for a suitable integer $m_{l}$. We shall define elements $\left\{u_{i}\right\}_{i=1}^{\infty}$ of $R^{*}$ inductively as follows: Let $u_{1}=\left(\gamma_{1}, x_{1}\right)$. For $u_{n}=\left(\gamma_{n}, \sum_{j=1}^{n} r_{j} x_{j}\right)$ $(r, \in K)$, let

$$
a_{1}=\left\{\gamma_{n}-\sigma_{J}\left(\gamma_{n}\right)\right\}^{-1}\left\{\gamma_{n+1}-\sigma_{J}\left(\gamma_{n+1}\right)\right\} r_{J} \quad(1 \leq j \leq n)
$$

and

$$
u_{n+1}=\left(\gamma_{n+1}, \sum_{l=1}^{n} a_{j} x_{j}+x_{n+1}\right) .
$$

Then it is easy to check that $o\left(u_{t}\right)=p^{r_{1}}-1$ and $u_{t}=u_{1+1}^{m_{t}}$. Let $f_{t}: G F\left(p^{r_{1}}\right)^{*} \rightarrow R^{*}$ be defined by $\gamma_{i}^{\prime} \mapsto u_{i}^{t}(t \in \mathbf{Z})$. Since $\left.f_{i}\right|_{G F\left(p_{p}^{\prime}\right) f^{*}}=f_{J}$ for $j \leq i$, there exists $f=\lim _{\rightarrow} f_{i}: K^{*} \rightarrow R^{*}$. As $f$ is a right inverse of $\pi^{*}=\left.\pi\right|_{R^{*}}: R^{*} \rightarrow K^{*}$, so $S_{1}=\left\langle f\left(K^{*}\right)\right\rangle$ is a coefficient subring of $R$.

We shall show that $S_{1}$ and $S$ are not conjugate in $R$. Let us suppose that there exists an element $v=\left(s, \Sigma_{1} d_{1} x_{t}\right) \in R^{*}\left(s \in K^{*}, d_{1} \in K\right)$ such that $S_{1}=v^{-1} S v$. Then, for each $i \geq 1$, there exists some $b_{\imath} \in K^{*}$ such that $f\left(\gamma_{\imath}\right)=v^{-1}\left(b_{\imath}, 0\right) v$. Then,

$$
\begin{aligned}
& u_{\imath}=\left(\gamma_{1}, \sum_{j=1}^{\prime-1} r_{j}^{\prime} x_{j}+x_{\imath}\right)\left(r_{j}^{\prime} \in K\right) \\
& =v^{-1}\left(b_{\imath}, 0\right) v \\
& =\left(s^{-1},-s^{-1}\left(\sum_{t} d_{t} x_{t}\right) s^{-1}\right)\left(b_{t}, 0\right)\left(s, \sum_{t} d_{t} x_{t}\right) \\
& =\left(b_{l}, \Sigma_{J=1}^{\prime}\left(s^{-1} b_{l} d_{J}-s^{-1} d_{J} \sigma_{J}\left(b_{l}\right)\right) x_{J}\right),
\end{aligned}
$$

which yields

$$
1=s^{-1}\left\{b_{t}-\sigma_{t}\left(b_{t}\right)\right\} d_{t} .
$$

So, for any $i \geq 1$, we see $d_{l} \neq 0$. This contradicts that $\sum_{l} d_{t} x_{i}$ is an element of the direct $\operatorname{sum} V=\bigoplus_{i=1}^{\infty} K x_{i}$.

In conclusion, we shall state a theorem which is a generalization of [3, Theorem].

THEOREM 4.1. (cf [9, p. 376, Theorem XIX.5] and [4, p. 491, Theorem 72.19]) Let $R$ be a ring with 1 . Assume that $J(R)$ is nilpotent. Let

$$
R / J(R)=\left(K_{1}\right)_{n_{1} \times n_{1}} \oplus\left(K_{2}\right)_{n_{2} \times n_{2}} \oplus \ldots \oplus\left(K_{d}\right)_{n_{d} \times n_{d}},
$$

where each $K_{l}(1 \leq i \leq d)$ is a commutative field of characteristic $p$ ( $p$ a prime) which is algebraic over $G F(p)$. Then there exists a subring $T$ of $R$ which satisfies the following. 
(i) $R=T \oplus N$ (as abelian groups), where $N$ is an additive subgroup of $R$.

(ii) $T$ is isomorphic to a finite direct sum of matrix rings over IG-rings.

(iii) $J(T)=T \cap J(R)=p T$.

(iv) $T / p T$ is naturally isomorphic to $R / J(R)$.

Moreover,, if $T^{\prime}$ is another subring of $R$ satisfying (ii)-(iv), then $T^{\prime}$ is isomorphic to $T$.

PROOF. Let $\bar{R}=R / J(R)=\bar{R} \bar{e}_{1} \oplus \bar{R} \bar{e}_{2} \oplus \ldots \oplus \bar{R} \bar{e}_{d}$, where each $\bar{R} \bar{e}_{1}(1 \leq i \leq d)$ is a simple component of $\bar{R}$ and $\bar{e}_{1}$ is a central idempotent of $\bar{R}$. Let $\bar{R} \bar{e}_{\imath}=\left(K_{l}\right)_{n, \times n_{1}}$, where $K_{l}$ is a commutative field which is algebraic over $G F(p)$. Let $\pi: R \rightarrow \bar{R}$ be the natural homomorphism. There are mutually orthogonal idempotents $e_{1}, e_{2}, \ldots, e_{d}$ of $R$ such that $e_{1}+e_{2}+\ldots+e_{d}=1$ and $\pi\left(e_{t}\right)=\bar{e}_{l}(1 \leq i \leq d)$. Then,

$$
R=e_{1} R e_{1} \oplus e_{2} R e_{2} \oplus \ldots \oplus e_{d} R e_{d} \oplus\left(\oplus_{1 \neq j} e_{1} R e_{j}\right)
$$

as abelian groups. Since each $e_{t} R e_{t}$ is semiperfect and $e_{t} R e_{\imath} / J\left(e_{\imath} R e_{\imath}\right) \cong \bar{R} \bar{e}_{\imath}=\left(K_{l}\right)_{n, \times n_{t}}$, there exist a local ring $S_{1}$ and an isomorphism $\phi_{1}$ of $e_{1} R e_{1}$ onto $\left(S_{1}\right)_{n_{1} \times n_{1}}$ (see, for instance, [1, p. 160, Theorem 21]). Let

$$
\begin{aligned}
& \phi=\phi_{1}+\phi_{2}+\ldots+\phi_{d}: e_{1} R e_{1} \oplus e_{2} R e_{2} \oplus \ldots \oplus e_{d} R e_{d} \rightarrow \\
& A=\left(S_{1}\right)_{n_{1} \times n_{1}} \oplus\left(S_{2}\right)_{n_{2} \times n_{2}} \oplus \ldots \oplus\left(S_{d}\right)_{n_{d} \times n_{d}}
\end{aligned}
$$

be the isomorphism. Since $S_{l} / J\left(S_{l}\right) \cong K_{l}$, by Theorem 2.2 and Theorem 2.3 (I), there exist an IG-subring $T_{1}$ and a left $T_{\imath}$-submodule $N_{\imath}$ of $S_{\imath}$ such that $S_{\imath}=T_{\imath} \oplus N_{\imath}$ (as abelian groups), and $T_{\imath} / p T_{\imath}$ is naturally isomorphic to $S_{\imath} / J\left(S_{l}\right)$. Then

$$
B=\left(T_{1}\right)_{n_{1} \times n_{1}} \oplus\left(T_{2}\right)_{n_{2} \times n_{2}} \oplus \ldots \oplus\left(T_{d}\right)_{n_{d} \times n_{d}}
$$

is a subring of $A$. Let $T=\phi^{-1}(B)$. As $J\left(e_{1} R e_{i}\right) \cap \phi^{-1}\left(\left(T_{i}\right)_{n_{i} \times n_{i}}\right)=J\left(\phi^{-1}\left(\left(T_{i}\right)_{n_{i} \times n_{i}}\right)\right)$, we see $J(t)=T \cap J(R)=p T$ and that $T / p T$ is naturally isomorphic to

$$
\left(e_{1} R e_{1} \oplus e_{2} R e_{2} \oplus \ldots \oplus e_{d} R e_{d}\right) / J\left(e_{1} R e_{1} \oplus e_{2} R e_{2} \oplus \ldots \oplus e_{d} R e_{d}\right)=R / J(R) .
$$

Let us put

$$
N=\phi^{-1}\left\{\left(N_{1}\right)_{n_{1} \times n_{1}} \oplus\left(N_{2}\right)_{n_{2} \times n_{2}} \oplus \ldots \oplus\left(N_{d}\right)_{n_{d} \times n_{d}}\right\} \oplus\left\{\oplus_{1 \neq j} e_{t} R e_{J}\right\} .
$$

Then we see $R=T \oplus N$.

Now, let us suppose that $T^{\prime}$ is a subring of $R$ satisfying (ii)-(iv). Let $e$ and $f$ be primitive idempotents of $T^{\prime}$. We claim that $R e \cong R f$ (as left $R$-modules) if and only if $T^{\prime} e \cong T^{\prime} f$ (as left $T^{\prime}$-modules). Let $\pi(e)=\bar{e}$ and $\pi(f)=\bar{f}$. Assume that $R e \cong R^{\prime} f$. Then $\bar{R} \bar{e} \cong \bar{R} \bar{f}$ as left $\bar{R}$-modules. Both $\bar{R} \bar{e}$ and $\bar{R} \bar{f}$ are minimal left ideals of $\bar{R}$, so they are contained in the same simple component of $\bar{R}$, which implies that $J(R)$ does not include $e R f$. Conversely, if $J(R)$ does not include $e R f$, then $\bar{R} \bar{e} \cong \bar{R} \bar{f}$, which means $R e \cong R f$ (see, for instance, [1, p. 158, Theorem 16]). Thus we see that $R e \cong R f$ (as left $R$-modules) if and only if $J(R)$ does not include $e R f$. Similarly, $T^{\prime} e \cong T^{\prime} f$ (as left $T^{\prime}$-modules) if and only if $J\left(T^{\prime}\right)=p T^{\prime}$ does not include $e T^{\prime} f$. Since $T^{\prime} / p T^{\prime}$ is naturally isomorphic to $R / J(R), J(R)$ include $e R f$ if and only if $p T^{\prime}$ includes $e T^{\prime} f$. So we see that $R e \cong R f$ (as left $R$-modules) if and only if $T^{\prime} e \cong T^{\prime} f$ (as left $T^{\prime}$-modules).

By making use of matrix units, 1 of $R$ is written in $T$ as

$$
1=\left(e_{11}+e_{12}+\ldots+e_{1 n_{1}}\right)+\left(e_{21}+e_{22}+\ldots+e_{2 n_{2}}\right)+\ldots+\left(e_{d 1}+e_{d 2}+\ldots+e_{d n_{d}}\right),
$$

where $e_{k l}$ are mutually orthogonal primitive idempotents of $T$, and $T e_{k l} \cong T e_{l}$ (as left $T$-modules) if and 
only if $k=l$. Similarly,

$$
1=\left(f_{11}+f_{12}+\ldots+f_{1 m_{1}}\right)+\left(f_{21}+f_{22}+\ldots+f_{2 m_{2}}\right)+\ldots+\left(f_{d 1}+f_{d 2}+\ldots+f_{d m_{d}}\right),
$$

where $f_{k,}$ are mutually orthogonal primitive idempotents of $T^{\prime}$, and $T^{\prime} f_{k_{1}} \cong T^{\prime} f_{l_{1}}$ (as left $T^{\prime}$-modules) if and only if $k=l$.

As $e_{k_{l}} T e_{k_{1}} / p e_{k_{1}} T e_{k_{l}} \cong e_{k_{l}} R e_{k_{l}} / e_{k_{l}} J(R) e_{k_{1}}$, we see that $e_{k_{l}}$ and $f_{l l}$ are primitive idempotents of $R$. Then $R=\oplus R e_{k l}=\oplus R f_{l}$ are indecomposable decompositions.

By what was stated above, Krull-Schmidt theorem tells us that there exists a permutation $\sigma$ of $\{1,2, \ldots, d\}$ such that $n_{t}=m_{\sigma(l)}$ and $R e_{t h} \cong R f_{\sigma(l) l}$ as left $R$-modules $\left(1 \leq i \leq d, 1 \leq k, l \leq n_{t}\right)$. By renumbering, we may assume $n_{\imath}=m_{\imath}$ and $R e_{\imath h} \cong R f_{\imath l}\left(1 \leq i \leq d, 1 \leq k, l \leq n_{\imath}\right)$. Now,

$$
T \cong\left(e_{11} T e_{11}\right)_{n_{1} \times n_{1}} \oplus\left(e_{21} T e_{21}\right)_{n_{2} \times n_{2}} \oplus \ldots \oplus\left(e_{d 1} T e_{d 1}\right)_{n_{d} \times n_{d}}
$$

and

$$
T^{\prime} \cong\left(f_{11} T^{\prime} f_{11}\right)_{n_{1} \times n_{1}} \oplus\left(f_{21} T^{\prime} f_{21}\right)_{n_{2} \times n_{2}} \oplus \ldots \oplus\left(f_{d 1} T^{\prime} f_{d 1}\right)_{n_{d} \times n_{d}},
$$

where $e_{11} T e_{11}$ and $f_{j 1} T^{\prime} f_{j 1}$ are IG-rings. Hence, to complete the proof it will suffice to show $e_{11} T e_{11} \cong f_{11} T^{\prime} f_{11}$.

As $e_{t 1} T e_{t 1}$ is an IG-ring which is naturally isomorphic to $e_{t 1} R e_{t 1} / e_{11} J(R) e_{11}$, so $e_{t 1} T e_{t 1}$ is a coefficient subring of $e_{11} R e_{11}$. Similarly, $f_{11} T^{\prime} f_{11}$ is a coefficient subring of $f_{11} R f_{11}$. As $\left.e_{11} R e_{11} \cong \operatorname{End}_{R} R e_{t 1}\right) \cong \operatorname{End}\left({ }_{R} R f_{11}\right) \cong f_{11} R f_{11}$, we see $e_{t 1} T e_{t 1} \cong f_{11} T^{\prime} f_{t 1}$ by Theorem 2.3 (II).

Note. It is unknown when the subring $T$ of Theorem 4.1 is unique up to inner automorphism of $R$ (see [3, Problem].

ACKNOWLEDGEMENT. The author would like to express his indebtedness and gratitude to Prof. Y. Hirano and Dr. H. Komatsu for their helpful suggestions and valuable comments.

\section{REFERENCES}

[1] BEHRENS, E. A., Ring Theory, Academic Press (1972).

[2] BOURBAKI, N., Éléments de Mathématique, Algèbre, Hermann (1962).

[3] CLARK, W. E., A coefficient ring for finite noncommutative rings, Proc. Amer. Math. Soc. 33 (1972), 25-28.

[4] CURTIS, C. W. \& REINDER, I., Representation Theory of Finite Groups and Associative Algebras, Pure \& Appl. Math. Ser. 11, Wiley-Interscience (1962).

[5] HASSE, H., Zahlentheorie, 2, Auflage, Akademie-Verlag (Berlin, 1963).

[6] JANUSZ, G. T., Separable algebras over commutative rings, Trans. A. M. S. 122 (1966), 461-479.

[7] JACOBSON, N., Lectures in Abstract Algebra, vol. III, Van Nostrand (1964).

[8] KRULL, W., Algebraische Theorie der Ringe, I, II and III, Math. Annalen 88 (1923), 80-122, 91 (1924), 1-46 and 92 (1924), 183-213.

[9] MCDONALD, B. R., Finite Rings with Identity, Pure \& Appl. Math. Ser. 28, Marcel Dekker (1974).

[10] MOTOSE, K. \& TOMINAGA, H., Group rings with nilpotent unit groups, Math. J. Okayama Univ. 14 (1969), 43-46.

[11] NAGATA, M., Local Rings, Wiley-Interscience (1962).

[12] RAGHAVENDRAN, R., Finite associative rings, Compositio Math. 21 (1969), 195-229.

[13] ROBINSON, D. J. S., A Course in the Theory of Groups, Springer-Verlag (1982).

[14] ROQUETTE, P., Abspaltung des Radikals in vollständigen lokalen Ringen, Abh. Math. Sem. Univ. Hamburg 23 (1959), 75-113.

[15] SERRE, J. P., Local Fields, Springer-Verlag (1979). 
[16] SUMIYAMA, T., On double homothetisms of rings and local rings with finite residue fields, Math. J. Okayama Univ. 33 (1991), 13-20.

[17] WILSON, R. S., Representations of finite rings, Pacific J. Math. 53 (1974), 643-649. 


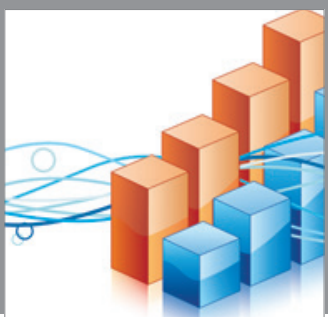

Advances in

Operations Research

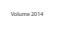

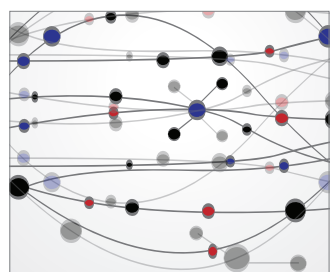

\section{The Scientific} World Journal
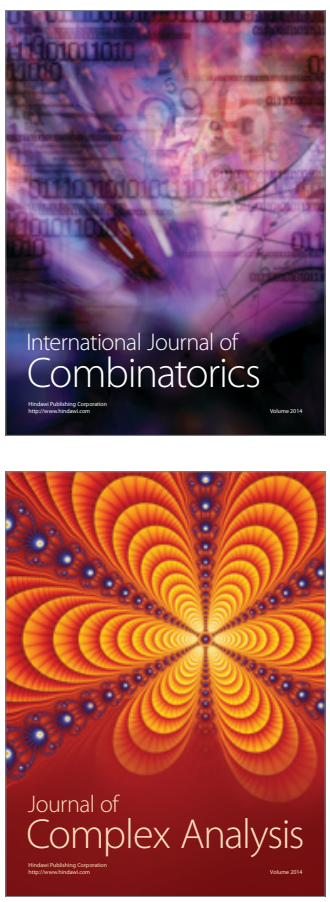

International Journal of

Mathematics and

Mathematical

Sciences
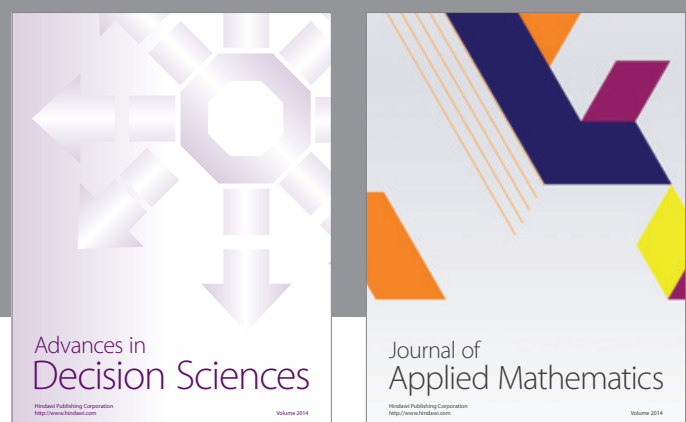

Journal of

Applied Mathematics
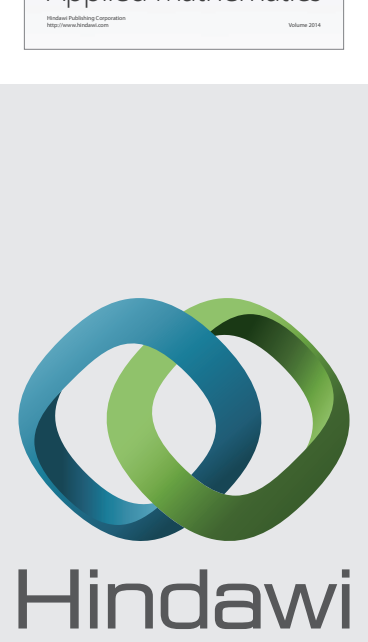

Submit your manuscripts at http://www.hindawi.com
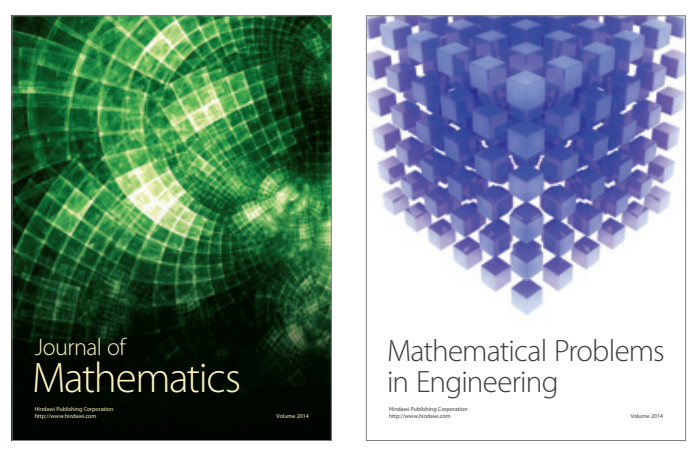

Mathematical Problems in Engineering
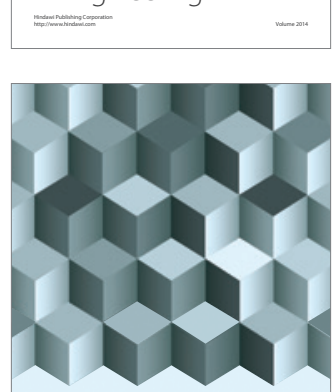

Journal of

Function Spaces
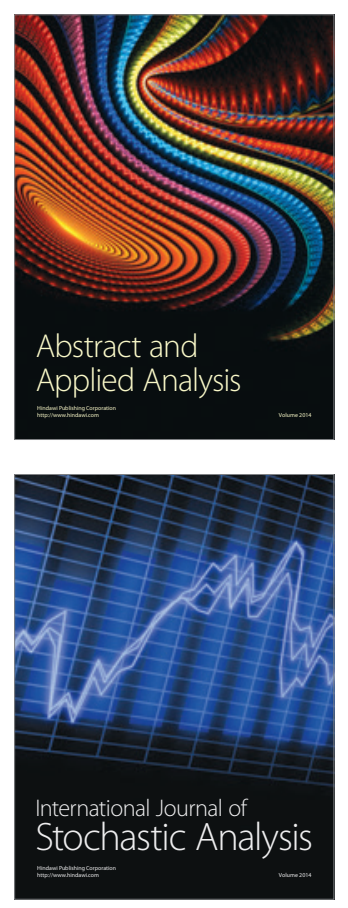

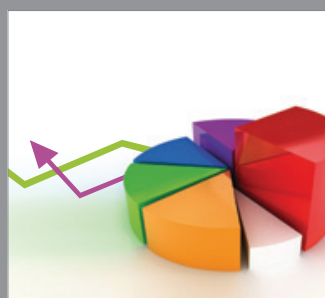

ournal of

Probability and Statistics

Promensencen
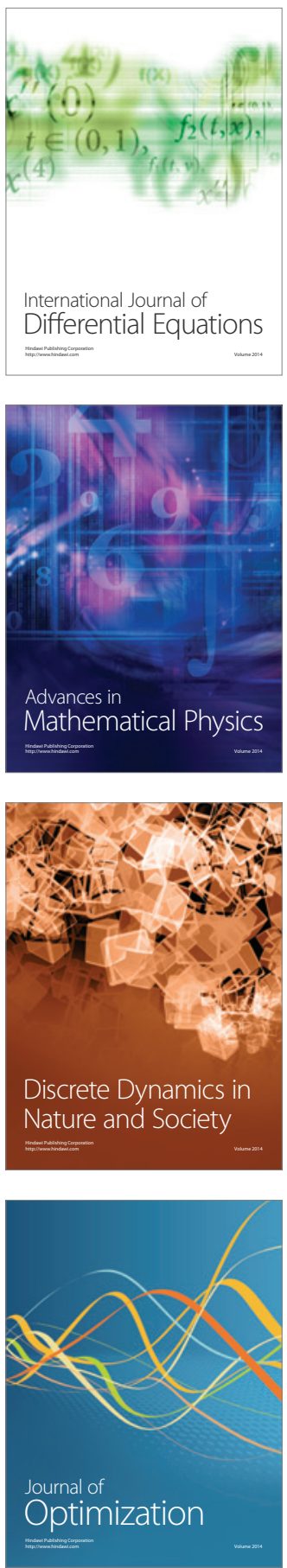